PROF. NATALIE PATTISON (Orcid ID : 0000-0002-6771-8733)

Article type : Original Article

\title{
Managing clinical uncertainty: an ethnographic study of the impact of critical care outreach on end-of-life transitions in ward-based critically ill patients with a life-limiting illness
}

\author{
Authors \\ Natalie Pattison*1,2,3 DNSc, MSc BSc (hons) RN n.pattison@herts.ac.uk; natalie.pattison@nhs.net \\ Jude Mclellan MSc RN jude.mclellan@christie.nhs.uk \\ Lara Roskelly³ MSc RGN lara.roskelly@rmh.nhs.uk \\ Kirsty Ainsworth ${ }^{4}$ MSc RN Kirsty.aisnworth@christie.nhs.uk \\ Theresa Wiseman ${ }^{3,5}$ PhD BSc (hons) RGN Theresa.wiseman@rmh.nhs.uk \\ *Corresponding author
}

Affiliations

1. University of Hertfordshire (present address) College Lane Campus, Hatfield, AL109AB

2. East/North Herts NHS Trust (present address) Lister Hospital, Corey Mills Lane, Stevenage SG14AB

3. The Royal Marsden NHS Foundation Trust, Fulham Road, London SW36JJ

4. The Christie NHS Foundation Trust, Wilmslow Rd, Manchester M20 4BX

5. University of Southampton, University Road, Southampton SO17 1BJ

This research was part funded by a research grant from the General Nursing Council Trust

This article has been accepted for publication and undergone full peer review but has not been through the copyediting, typesetting, pagination and proofreading process, which may lead to differences between this version and the Version of Record. Please cite this article as doi:

10.1111/jocn.14618

This article is protected by copyright. All rights reserved. 
Abstract:

Rapid response teams, such as critical care outreach teams, have prominent roles in managing end-of-life transitions in critical illness, often questioning appropriateness of treatment escalation. Clinical uncertainty presents clinicians with dilemmas in how and when to escalate or de-escalate treatment.

Aim: To explore how critical care outreach team decision-making processes affect the management of transition points for critically ill, ward-based patients with a life-limiting illness.

Methods:

An ethnographic study across two hospitals observed transition points and decisions to de-escalate treatment, through the lens of critical care outreach. In-depth interviews were carried out to elucidate rationales for practices witnessed in observations. Detailed field notes were taken and placed in a descriptive account. Ethnographic data were analysed, categorised and organised into themes using thematic analysis.

Findings:

Data were collected over 74-weeks, encompassing 32 observation periods with 20 staff, totalling more than 150 hours. Ten formal staff interviews and 20 informal staff interviews were undertaken. Three main themes emerged: early decision-making and the role of critical care outreach; communicating end-oflife transitions; end-of-life care and the input of critical care outreach. Findings suggest there is a negotiation to achieve smooth transitions for individual patients, between critical care outreach, and parent or ward medical teams. This process of negotiation is subject to many factors that either hinder or facilitate timely transitions.

This article is protected by copyright. All rights reserved. 


\section{Conclusions:}

Critical care outreach teams have an important role in shared decision-making. Associated emotional costs relate to conflict with parent medical teams, and working as lone practitioners. The cultural contexts in which teams work have a significant effect on their interactions and agency.

Relevance to practice: There needs to be a cultural shift towards early and open discussion of treatment goals and limitations of medical treatment, particularly when facing serious illness. With training and competencies, outreach nurses are well placed to facilitate these discussions.

Keywords: Critical Care Outreach, End-of-Life Decision-Making, Ethnography

\section{Background}

Critical illness episodes during the course of life-limiting illness will often lead to transitions in patients' care. Transitions in care, points at which the trajectory and goals of treatment change, involve a limitation or de-escalation of medical treatment. At this point, intentions of medical treatment are questioned and can change from curative to palliative in patients with acute pathophysiological deterioration. This process can be difficult for patients, family and clinicians to manage. Critical care outreach teams (CCOT), also referred to as rapid response and medical emergency teams (MET), have a prominent role in triaging sick, at-risk or deteriorating patients for admission to critical care, and provide care for these patients on the ward.

For many patients, the first time a limitation of medical treatment (LOMT) decision is made, such as 'not for admission to critical care', will be when they become acutely unwell. At this point, clinicians examine prognoses for underlying illnesses, consider potential prognosis from their acute illness and

This article is protected by copyright. All rights reserved. 
explore possible treatment options. These critical transition decisions have significant consequences for smooth transitions in foci of care. Uncertainty in decisions appears to hinder smooth, timely and sensitive transitions for patients and families (Fisher and Ridley, 2012; Pattison et al, 2013). Decisions to limit critical care treatment, or not admit to critical care, can mean that a person's disease trajectory is shortened, and death may be perceived to occur more quickly (Fisher and Ridley, 2012; Hua et al 2016; Dahmen et al 2017). Conversely, while admitting to critical care might, but not always, lead to prolonging life, it can also mean subjecting a person to difficult and painful treatments for uncertain benefit (Aslakson et al 2015; Pattison et al 2013). Critical care outreach play a key role in deciding to escalate, or de-escalate care, since it is most often at the point of serious and acute illness that a review of goals of treatment is made (Jones et al 2012; Pattison et al 2015). One systematic review emphasized that LOMT decision-making constituted up to a third of medical emergency team (MET) calls and LOMTs occurred more frequently than resuscitation interventions such as endotracheal intubation (Tan and Delaney, 2014). Many patients in these studies undergoing LOMTs had a cancer diagnosis (Tan and Delaney, 2014; Coombs et al 2016), highlighting that this patient group in particular encounter difficulties in decision-making and poor care as a consequence (NCEPOD, 2008; Hui et al 2012; Bennet et al 2016). Clinical uncertainty refers to where doubt about prognostic accuracy affects and delays decision-making about treatment (Ridley and Fisher, 2012).

Research demonstrates that outreach teams often lead discussions of limitations of treatment (subsequently leading to end-of-life decision-making) (Calzavacca et al, 2010; Pattison et al, 2010; Jones et al, 2012; Tan and Delaney, 2014; Coombs et al 2016). Frequently, decisions about continuing treatment are addressed only when patients become critically or acutely ill (National Confidential Enquiry Peri-Operative Deaths [NCEPOD], 2008). In critical illness, patient-centredness, deemed a priority area by the UK government (Department of Health, 2009), can lose focus and

This article is protected by copyright. All rights reserved. 
survival takes priority (Frost et al, 2011), resulting in timely opportunities for end of life (EOL) transitions being lost (Jones et al, 2012; Pattison et al 2015). The ensuing consequences are that good and timely EOL care are less likely due to missed opportunities and patients being in receipt of life-sustaining interventions rather than the focus being on comfort care, and that dying patients receive unnecessary interventions. This is evidenced by a large review of 1.2 million admissions where the mean number of admissions in the last 12 months of life averaged 2.28 , leading to 30.05 bed days occupied (Bardsley et al, 2016).

Critical illness, related or unrelated to underlying disease pathology, creates further uncertainty for clinicians because there are unknown elements in disease processes and prognostication (Bristowe et al, 2015; Pattison et al, 2015). Previous ethnographic work in the critical care unit suggests that there are debilitating emotional consequences for those involved in decisions (Seymour, 1999), especially where conflict arises (Higginson et al, 2015). These critically ill, deteriorating patients at risks of dying were not discharged home and over half died in hospital. These figures highlight the lack of advance care planning (ACP), advocated by the UK government (Department of Health, 2009; Gold Standards Framework, 2018); as a result, care preferences may not reflect those of families or patients. The role that these rapid response teams, such as MET and CCOT, have in EOL decisionmaking highlights challenges in terms of decision-making leadership and autonomy to challenge (Calzavacca et al, 2010; Tan and Delaney, 2014; Pattison et al, 2015; Bennet et al, 2016). Despite exponential worldwide growth in these teams, little guidance exists for CCOTs and METs about dealing with transition points in life-limiting illness. Transition points in care as described in this work refer to transitions to escalation of care or limitation or de-escalation of medical treatment.

This article is protected by copyright. All rights reserved. 


\section{Primary aim}

To explore how critical care outreach team decision-making affects the management of transition points for critically ill ward-based patients with a life-limiting illness.

\section{Methods}

\section{Design}

This study involved an ethnography of the critical episodes to establish factors (facilitating and/or inhibitory) and the experience of critical care outreach for improving these transitions in patients with cancer.

\section{Sample and Setting}

Participants included: staff caring for patients with life-limiting illnesses, across two hospitals on three sites. Site 1 and 2 were part of a specialist cancer centre in the South of England. Site 3 was a specialist cancer centre in the North of England. A purposive sampling approach of observation episodes across at least twenty episodes was deemed to be sufficient in order to generate ethnographic data (Hammersley and Atkinson, 2007).

\section{Data collection}

An ethnographic approach was undertaken incorporating participant observation (PO), interviews and questionnaires, involving observation of critical transition points. These transition points

This article is protected by copyright. All rights reserved. 
included observation of: points at which patients were reviewed at the bedside, office-based discussions, referrals from teams (face-to-face and telephone), and bedside discussions with patients and families regarding possible treatment options. Data collection involved both set days for observation, which were scheduled (site 1,2,3), and an 'on-call' approach (site 2 and 3), where teams called the researcher when there were likely to be transitions to observe. This reflected the reactionary response model that CCOT work in; they are called when patients are acutely deteriorating. Ethnography requires the researcher to observe the natural setting (i.e. the ward, as well as CCOT/medical team offices), in order to give meaning to the context in which situations occur (Hammersley and Atkinson, 2007). In this study, the 'privileged observer' role was undertaken, whereby the researcher observed transition points (in a helper role) with patients attended by critical care outreach. Access to the teams was gained via the hospitals' CCOTs and METs (via multidisciplinary teams) managers. For each observation, $\left[{ }^{* *}\right]$ observed discussions and actions related to transition points and noted the subsequent outcomes for patients. Detailed field notes were taken during and/or after observation, in an attempt to capture all the relevant data and narratives for each episode witnessed and place it in a descriptive account, accumulating over time to a 'corpus' (Emerson et al, 2001). Informal (annotated, rather than formal audio-recorded) and formal interviews were conducted after observations, in order to develop understanding of situations that were observed and decision-making in relation to those situations (Hammersley and Atkinson, 2007). A core interview guide was developed to inform key questions regarding decision-making and process.

\section{Ethics}

The study was given a favourable ethical opinion by an NHS Research Ethics Committee (REC ref: 13/LO/0744) and informed, written consent was taken from participants. Specifically, patients were informed of the study and written consent was obtained from staff, the key informants. Patients and

This article is protected by copyright. All rights reserved. 
families were involved in the design of the study via an institutional Patient and Carer Research Review Panel. Ethical considerations centred on the PO role and the sensitivity required in observing clinicians' practice with critically ill ward patients and families.

Interviews

In-depth interviews were conducted by [**]. Both formal interviewing (audio-recorded and interview cues used) and informal interviewing, using conversational interviewing techniques and annotated, were carried out to elucidate rationales for practices witnessed in the participant observation phase, encompassing: decision-making, factors (facilitating and/or inhibitory) around managing transitions, and characterization of practices around uncertainty in life-limiting illness. This helped enhance the PO by allowing clarification of any complex issues or confusion that arises during PO, and add depth to the overall data (Emerson et al, 2001; Higginson et al, 2015).

\section{Analysis}

Qualitative data from ethnographic participant observation field notes, documents (medical notes if needed) and interviews were analysed using an inductive-iterative approach to analysis and aided by reflexive notes. In regards to managing the tensions in the PO role, reflexive diaries, and regular supervision with an experienced ethnographer aided data collection and analysis (Savage and Moore, 2004; Hammersley and Atkinson, 2007). Data were analysed using cross-case comparative analysis, across each case and each type of data (interviews/ observations), was carried out, with inductive analysis techniques based on thematic analysis to develop themes, based on observations (Bloor, 1978). An initial thematic list was derived (based on 146 codes) and refined to reach the final themes. Eventually, from this domain analyses and taxonomies were developed, outlining and

This article is protected by copyright. All rights reserved. 
describing cultural themes in a final ethnographic account (Hammersely and Atkinson, 2007). All data and analysis were reviewed by an experienced ethnographic researcher in order to enhance dependability and credibility. Credibility was addressed by presenting believable accounts and constructs of the work (Lincoln and Guba, 1985) and dependability through constructing interpretation was constructed to try and avoid instability (Lincoln and Guba, 1985). Supervision and reflection were important for ensuring both these elements, as was confirmability (Lincoln and Guba, 1985), the audit and data trail maintained. As per ethnographic data analysis, themes were derived from both observational and interview data, rather than these being regarded separately.

Data were collected over 74 weeks from October 2013 to January 2015 at Sites 1 and 2, and for 51 weeks from December 2013 to December 2014 at Site 3. Thirty-two observations were carried out, with 20 CCOT nurses, resulting in over 130 hours of observation data, across the two hospitals (on three sites). Participant characteristics are outlined in Supplementary file 2. Twenty informal interviews and ten formal audio-recorded interviews were conducted.

\section{Contextual description of practice}

Critical care outreach teams (CCOT) in the three sites worked seven days a week, with 12-24 hour a day coverage. Some teams did not cover the night shift period at the time of data collection. All CCOT members were nurses and were independent of critical care units. Shifts ranged from 12 hours to 8 hours (twilight shift to cover the evening period) and there was a handover period of half an hour to allow for discussion of cases. This was often used as an informal opportunity for debriefing. Staff most often worked alone on a shift; however junior staff would sometimes be assigned a more senior staff member to work alongside. The role of CCOT was to identify and support ward-

This article is protected by copyright. All rights reserved. 
based deteriorating and critically ill patients on the ward, assessing appropriateness for, and facilitating or preventing admission to critical care (Department of Health, 2000).

\section{Findings (Results)}

\section{Overview of Themes}

Three main themes and eight sub-themes emerged from both the interview and observational data of transition points, considered together as an ethnographic account: Early decision-making and the role of critical care outreach; Communicating end-of-life transitions; End-of-life care and the input of CCOT. These encompassed three broad levels of influence: the micro, practice at a local and individual level, meso the practice or events within a hospital or team culture, and macro, issues and events that related to broader professional or health service culture. These domains are represented in table 1 below. The supplementary file (Table 2) outlines observational and interview data excerpts for each theme and sub-theme.

The ethnography outlines the tensions encountered by CCOT in trying to facilitate timely decisions, resulting in proportionate levels of care and treatment, but also the influence on this transition on their own beliefs; and their tangible impact on decisions made. Figure 1 presents the interrelationships of the sub-themes and the levels of interaction as a diagram. The larger area of triangles emphasises the macro elements (organisational), the middle area the meso (the team level issues) and the smaller area towards the point of the triangles, the micro (individual level).

This article is protected by copyright. All rights reserved. 


\section{The role of critical care outreach in early decision-making}

CCOT appeared to have an important role in precipitating early decision-making. The timing of decisions was affected by clinical uncertainty, contextual factors such as CCOT workload and team structures, and the influence of power on the process of reaching decisions about transitions.

CCOT were called to provide ward-based support to critically ill patients, and escalate, or not, the patients' transfer to critical care. However, the perception of the CCOT nurses' role was not always clear. Some consultants seemed to perceive that CCOT should only be there to provide critical care support, and not question intent of treatment, which was at odds with how the CCOT nurses viewed their roles. There seemed to be an undercurrent of CCOT intervention being a perceived threat to medically-led decision-making, emphasizing the interplay that power has, specifically in relation to professional roles (both nurses' and doctors' personal and intra-professional perceptions, as witnessed below) and the subsequent role CCOT have in directing treatment decisions.

Following a case where a patient's consciousness had decreased and their breathing had become laboured, with respirations around $6 / \mathrm{min}$, a 2222 call (cardiac arrest call) was made. CCOT arrived and it was established it wouldn't be appropriate to escalate treatment to critical care given the advanced nature of the patients disease and intercurrent illness, CCOT directed a clinical situation.

"The patient was very unwell and retaining carbon dioxide to the point it made him unconscious, with little prospect for reversibility and extensive disease. Discrete discussions led by CCOT led to agreement he was dying. Following those CCOT prompts, the Senior House Officer (junior doctor) called the registrar and agreed to change from level two down to ward-based care only and for referral urgently to palliative care, so we handed over the patient to her. (Participant Observation field notes, site 3, Mar 14)

This article is protected by copyright. All rights reserved. 
In this situation, CCOT direction was not questioned and the transition for the patient appeared smooth. However, in other observations this was not evident.

"Having been called to review a patient, known to CCOT and with extensive medical and surgical history, who was deteriorating on the ward, the CCOT nurse mentioned to the patient about the fact that she'd seek clarification regarding the overall plan. They had discussed plans previously regarding wishes for escalation of treatment and what further critical illness episodes could mean and how this would affect future plans for treatment. It was important to note that the consultant of this patient was away, and reportedly did not like delegating such decisions to his registrars [ senior doctors in training], ultimately meaning that no decisions could be made in his absence. When we went to discuss with the team doctors, they seemed very reluctant to engage. The patient's clinical nurse specialist, who we had also engaged agree a palliative care referral and transition to end of life would be appropriate. Despite the junior medical staff agreeing, they would not make any decisions, leading to an apparent sense of frustration for CCOT who had to support this patient through their deterioration despite no clear treatment goals." (Participant Observation field notes, Site 2, Mar 14)

This reluctance for CCOT involvement, unless escalation plans were unquestioned, appeared emblematic of a culture in certain areas of medical practice. Certain teams were reluctant to have early or timely conversations about goals of treatment, particularly when the consultant was absent, and it appeared that it was not until CCOT responded to calls to be involved that these conversations were initiated, either by the parent team or by CCOT.

This article is protected by copyright. All rights reserved. 
"We overheard a discussion at the nurses' station, between a junior doctor and ward nurse, regarding a patient who was not for resuscitation, but who was very poorly and it happened to be a patient we were due to see as they were on the CCOT 'list'. She was a lady who had brain metastases, secondary to her lung cancer, brittle and life-limiting asthma and now had a chest sepsis. She had a documented treatment escalation plan that her care would be limited at level two care. We drifted over to nurses' station and joined in the discussion; the junior doctor was new and not known to CCOT and there was a new system in place with an acute medical consultant for these kinds of patients. It appeared from discussion with the CCOT nurse that critical care outreach were much less involved in these kinds of patients and there was an allusion that outreach were perhaps seen as a threat. The matter was complicated due to the availability of the decision-maker, who was only there on certain days and he wasn't available that day. This led to apparent confusion about how much CCOT should get involved or not. (Participant observation field notes, Site 3, Dec 14)

The influence of individuals was important in this process; with particular consultants involved, or particular CCOT members facilitating or creating barriers to engagement and joint-working. As this nurse described:

"Some teams already have a plan in place and openly discuss that with you, but quite often we come up again hurdles where we have to instigate the discussion, which is often brushed under the carpet."

This article is protected by copyright. All rights reserved. 
Even when working within the confines of a responsive model, CCOT would manipulate practices to ensure their views were considered, challenging notions of parent medical teams holding the power in decision-making. Strategies to negotiate this role conflict included: discussing patients directly with palliative care teams, and particularly CCOT nurse to palliative care nurse, to gather support for the move to limit treatment or apply an escalation limitation.

Challenging power and contributing to decisions about end of life appeared to have emotional consequences for CCOT as individuals and teams. In some cases they built up relationships over several weeks, supporting around critical care admissions and then facilitating appropriate and proportionate decisions about treatments in the face of a new onset of critical illness. The emotional implications of this seemed challenging, especially where uncertainty was present, and it also appeared to affect confidence for some, especially junior CCOT staff, and this related to selfefficacy. On a meso and micro level, the lack of collegiate support or opportunity to debrief available meant that the CCOT nurse reported significant emotional burdens and consequences of precipitating timely EOL decisions alone. The lack of timely decisions, related to clinical uncertainty, also led to what was termed by CCOT as the '3am phenomenon', or the 'Friday afternoon nightmare'. Decisions were left, with the deteriorating patient in limbo, until a crisis point, such as a new onset of critical illness, was reached. Discussing a young 24-year old patient, under CCOT care on and off for weeks, and who was now dying, this CCOT nurse outlines the tensions experienced in working with different medical teams out-of-hours:

“...The team on the weekend had not actually ever met [the patient] He was not documented for limitations of care, he was for active everything. I discussed him with the new consultant and research reg[istrar], who'd never met him, and their aim was get him through the weekend. And to 
have to go in at that point and start talking about withdrawal at the point, when the family didn't know them would have been unfair. He was having no palliative input at that point."

[Formal interview CCOT Nurse 2, Site 1, June 14]

Clinical uncertainty seemed to pervade each aspect of work during transitions to EOL, and CCOT provided an opinion to help clarify uncertainty in relation to critical care and deterioration status. However, this uncertainty was heightened sometimes to the point where it impinged on appropriate decision-making. This appeared to be a deeply embedded cultural issue of fear of uncertainty, from both professional and institutional perspectives and this macro-level phenomenon had consequences at micro and meso levels. This is exemplified in an encounter with a patient with leukaemia, aged 50, and a serious heart condition in a peri-cardiac arrest situation. CCOT undertook various rescue interventions (e.g. catheterisation, fluid resuscitation, blood gas analysis), closely observing and monitoring the patient and further examination. The patient reported being utterly overwhelmed by consultations and professionals, and had not understood what the various teams had said, so CCOT explained the clinical implications.

"The patient asked how critical was critical, and [the CCOT nurse] explained the possibility of death as she outright asked if she was dying.. She had several other concomitant medical problems, so had been in frequent discussion with the parent medical team. CCOT seemed conscious that at the outset at referral questions were asked re: resus[citation] and overall escalation plan, and it appeared that all active treatment was the plan, following several discussions about what the right course of action should be."

[Participant observation field notes, Site 3, May 14]

This article is protected by copyright. All rights reserved. 
Indecision about whether to escalate and admit to critical care created difficulties around information-giving to the patient and uncertainty for the patient. However, clinical uncertainty was difficult to manage, especially where emotions were heightened because it was regarding an end-oflife or emergency situation.

On observation there seemed to be a macro-level phenomenon of buying time during uncertainty in critical illness by carrying out clinical interventions. This strategy was used either, to gather clinical cues and build a bigger picture to inform decision-making, or to understand if a condition was reversible. There appeared to be a limbo period when clarifying investigations were taking place, to establish a patient's condition, or verify their disease status; revealing what was going on. However, this seemed to limit EOL care planning opportunities; only planning discussion about what might happen if the investigation confirmed progressive cancer and that the patient was also recognised as dying.

An added layer of complexity related to uncertainty around diagnosing dying. It seemed that if presenting critical illness was the precipitating factor in a patient's decline, leading to discussion about goals of treatment, CCOT would often be heavily involved, either with the support of their critical care unit senior colleagues or with the teams. Some CCOT nurses would deal with uncertainty at the outset, questioning, upfront at point of referral, about limitations of care. Depending on the nurses' comfort at being able to do this, patient preferences might be sought, where appropriate:

"I'm from critical care outreach and we are here to see if we can help you get better and if you need critical care support. If you do need critical care support then this might mean taking you into the critical care unit, how do you feel about that?"

[Informal interview, CCOT nurse 17, Site 1, Feb '14]

This article is protected by copyright. All rights reserved. 
The parent medical teams' expectations about the level of care to be provided would also be established. Once their expectations had been clarified, CCOT might either challenge this, or moderate their own expectations of limitations of treatment and care levels. This was a constant process of negotiation. Out-of-hours treatment decisions seemed to create barriers to timely decision-making on each site, when patients and CCOT could be left in apparent limbo out-of-hours.

". . we attended a patient who collapsed with advanced cardiac disease on a Friday afternoon in outpatients, questions were immediately raised by CCOT as to what the overall plan was, since it was the weekend imminently. The medical team were reluctant to make a decision but we hung around for nearly 45 minutes in the doctors' office until a formal decision was agreed"

(Participant observation field notes, Site 3, Mar '14).

The stress of having to address decision-making issues out-of-hours, with less senior support became evident in many accounts and observations. CCOT's level of persistence in getting decisions made out of hours appeared to be mitigated by various strategies including: hanging around consultant offices, waiting in doctors' offices, and repeatedly calling through the ranks of doctors. Where decisions were unclear, CCOT remained as first point of contact for any acute emergency, appearing to cause them further moral dissonance; where they felt they could not act according to their moral values and had to provide life-sustaining therapy even where it was not perceived to be the appropriate course of action.

"We feel quite annoyed that the team have been aware for quite some time that they are not going to do [anything], and it's us responsible out of hours... It lands on our doors as the bad guys. It [decision-making] should have been done. . I felt awkward about treading on toes."

[Informal interview, CCOT Nurse 3, Site 1, Sep '14]

This article is protected by copyright. All rights reserved. 
The decision-making process regarding limitations of medical treatment often took place over several CCOT visits, a process of careful negotiation over time. It seemed as though, the seed would be sown with the parent medical teams, and at times, by gently establishing what the patients thought was going to happen, and then the issue would be revisited the next shift, or as the patient's condition changed. Expectations of the team would also be established, and either challenged or CCOT's own expectations of limitations of care would be moderated. A heuristic approach to decision-making from medical teams seemed to take place, whereby previous experiences with patients, and those patients' outcomes, had influenced their subsequent thinking and decisions. This contrasted with a more rational model approach from CCOT, whose decisions were based on their interpretations of patients' survival chances, or possibility for reversal of critical illness.

\section{Communicating end-of-life transitions}

This theme was underpinned by how CCOT appeared to view the institutional and team cultures of addressing EOL transitions and other team members' ability to communicate EOL issues, with patients, families and each other. It was characterized by individuals' ability to challenge, based on experience and personality, but also by the institutional, discipline and individual respect for CCOT.

"A new critical care doctor arrived at the scene, his first day in the hospital, and he tried to direct the emergency situation, asserting authority and I noticed how the rest of the people around the bed referred their questions to the CCOT nurse and not him. They were used to CCOT leading these situations, having done a lot of ward-based training with the CCOT team members. The CCOT nurse continued to lead, in a gently assertive way, reassuring the patient throughout."

(Participant observation field notes, Site 3, Feb '14)

This article is protected by copyright. All rights reserved. 
All of these factors affected the ability to deliver patient and family-centred care through open communication. The opportunities for CCOT nurses to escalate to senior medical colleagues, was reported as limited, and often they spoke to critical care consultants to try and help support their argument for or against escalation of treatment. Two approaches appeared to be present: a consultative model where parent medical teams (the teams ultimately regarded as responsible for treatment decisions, led by admitting consultant or primary team) sought advice, and engaged with CCOT recommendations and a responsive model, where parent medical teams appeared to engage with CCOT when recommendations aligned with their team's preferences. An example of the consultative model can be seen below, and also exemplifies the final theme and how CCOT input to EOL care:

"There was a call to CCOT about a patient who'd been 'on their books' [under CCOT review] for a few days and as the CCOT nurse described it - "it was really a case of [parent] 'team acopia'". There had been an intensive care unit admission for the patient in the preceding few days but the issue probably could have been managed on the wards, and would not normally necessitate intensive care unit admission but it was facilitated because the parent team were struggling. Recent results since this admission showed extensive Leukaemic progression so he wouldn't have been suitable for aggressive intervention or further cancer treatment. The teams came together on the ward and decided to go down the route of full palliation. However, he was on nasal high flow oxygen and quite dependent on it. CCOT were called on to help further manage the patient's transition towards end of life care, whilst on nasal high flow."

(Participant observation field notes, Site 1, June '14)

This article is protected by copyright. All rights reserved. 
An advocacy role (underpinned by knowledge as expert critical care practitioners) in relation to transitions, as another layer to this consultative approach, seemed to create tensions with some parent medical teams.

These tensions extended to junior doctors who also seemed to face strains in transcending knowledge-based domains of knowledge to actually communicating to patients about their clinical situation, in the context of medical hierarchy, where more senior clinicians are expected to discuss these issues.

"The SHOs [junior doctors] have said that the more senior you are, the more difficult it is to have those discussions, and we've on our team day been having training on how to break bad news, we are already doing this but it is to show that we are willing to take part in those conversations and then get the registrars and consultants involved, have there is frank and honest discussions when it is very, very obvious and nobody has broached the subject. You know: What are their wishes?. .".

[Formal interview, CCOT Nurse 15, site 2, June '14]

Although all sites were tertiary treatment centres, and thus used to treating people with advanced disease, there still appeared to be cultural acceptance of individual pockets of poor decision-making. The influence of macro phenomena, through organisational approaches, communication aides and hospital culture was reflected in documentation use. One site (site 3) used a prompt form regarding treatment escalation, and these forms acted as prompts for EOL discussions, and were placed in the front of patients' medical records. However, these forms were not regarded as a panacea. Conflict regarding EOL transitions, limitations of medical treatment and resuscitation decisions was still reported and observed, sometimes in relation to how appropriately forms were used. CCOT were

This article is protected by copyright. All rights reserved. 
observed pursuing teams to give greater detail and granularity to these decisions on the forms. CCOT sought a clear plan as they argued the consequences of unnecessary, inappropriate or disproportionate care could be catastrophic for families and patients, and CCOT, who experience untimely decisions, decision reversals and unnecessary resuscitation or treatment.

"They [parent teams] think because we're asking about escalation that we want patients to be made not for resus, that's not the case, we neither disagree or agree, we just want a plan in place. Because what we don't want is to be caught in the early hours of the morning, which happens quite a lot really... without a plan. They see it as - tunnel vision, they just see it that we want them made not for resus[citation]. [Formal interview, CCOT Nurse 10, Site 3, Dec '14]

They don't realise the situations that we find ourselves in, which we see it from all angles. It's traumatic for the patient and families particularly, but also the staff involved if there isn't a proper plan in place then it can have huge repercussions, and I don't think they always consider that." [Formal interview, CCOT Nurse 11, Site 3, Dec 14]

Interviews also emphasised how the transition was about so much more than documentation, and a Do Not Attempt Cardiopulmonary Resuscitation (DNACPR) form; it related to decisions about symptom control, addressing different eventualities or possibilities, communicating those appropriately to families and patients, establishing limitations of treatment, and at what point those might change. These nuances of EOL transitions and decision-making were hard to convey to teams, who saw it as more black and white in terms of DNACPR.

This article is protected by copyright. All rights reserved. 
". . .It's about how do you recognise a deteriorating patient and a dying patient. even from the intensive care side of things we are often managing a good death. It's about being able to recognise, we need a change of gear, because we see it at the coalface a lot more, so we're probably quite good at doing it. "

[Formal interview, CCOT Nurse 15, Site 2, June '14]

Following an implied procedure or hierarchy also seemed to be an issue in communication, when CCOT asked a doctor (senior registrar) about plans to escalate or limit treatment for a patient with a previous lengthy critical care unit (CCU) stay, and who was deteriorating on the ward again:

'The CCOT nurse asked, "My question to you is: if she deteriorates further respiratory-wise, would she [the patient] be for everything [for full escalation of treatment]?" The registrar responded that she couldn't make that decision and she'd have to get hold of her consultant. CCOT nurse politely asserted that "we'd need to know in order to make a plan for out of hours", and there appeared to be mutual respect. It emerged later that she hadn't managed to get hold of her, so it was left in limbo. The registrar explained if the time came [to a patient rapidly deteriorating to the point of new critical care interventions], we could talk to the patient then."

(Participant observation field notes, Site 1, June '14)

However, there was also an additional element of positive trust-building and improved communication that emerged as relationships were established between teams; when doctors knew CCOT and respected their reputation, they were willing to engage indepth rather than unwillingly or superficially. This again, was very individualistic, with self-

This article is protected by copyright. All rights reserved. 
efficacy seeming to play a part on a micro-level; confidence was important in communication around challenging situations. Frequent, short junior doctor rotations created reported difficulties for CCOT having to support these relatively inexperienced doctors in caring for sick patients on the ward. By the time it came to move on six months later, the cycle would begin again of building and establishing trust and rapport. The culture of respect appeared to be influenced by medics' positive experiences of CCOT, and also related to critical care teams' relationships with CCOT.

"Having been involved in a patient's care and treatment escalation in preceding days, the CCOT nurse was concerned about not having a plan of treatment for this deteriorating patient. We walked into the joint doctors/nurses office and the CCOT nurse asked who was looking after her that day and asked the outcome of yesterday's parent team discussions at MDT. The senior doctor seemed keen to talk to the CCOT nurse, and seemed to respect her decisions. He appeared engaged, and stood up to talk to us, listening to what the CCOT nurse had to say."

(Participant Observation Field Notes, Site 2, October'13)

Ease of access to the teams determined incidental discussions about end-of-life decisions, or limitations of medical treatment, and appeared to enhance communication.

\section{End-of-life care and the input of CCOT}

It was observed how CCOT also seemed to have a role with ward staff and families to help them to see the clinical cues of dying patients, so that timely EOL care could be initiated. It was characterized by the extent to which CCOT were involved beyond decision-making, through to supporting the EOL transition and providing elements of EOL and family care. It overlapped with team structure and the influence of being a lone practitioner was prominent; working with little support meant not all CCOT

This article is protected by copyright. All rights reserved. 
were willing or able to be involved beyond the decision-making, and the emergency care workload was a significant factor.

CCOT were active in provision of end-of-life care. They supported palliative care when end-of-life care involved difficult pharmacology, such as using anaesthetics/sedation, emphasising how they seemed to have developed a good working relationship with palliative care.

"The patient was not for active treatment just for palliative care, she had a high early warning score, a chest drain for pleural effusions, which was not draining, tachypnoeic, and an irregular heart rate. She had been referred by ward nurse and on looking at her noteswe saw a limitation of escalation of treatment form in the notes and was documented to receive only ward-based care. We had a discussion with the ward nurse, and actually patient probably didn't need to be seen other than to manage her nasal high flow oxygen on the ward. The ward nurse apologised for referring but CCOT emphasised that it did not matter, CCOT were there to help."

(Participant Observation Field Notes, Site 3, Feb '14)

Tensions appeared to exist between wanting to get involved in EOL patients' care, whom they had previously been involved with, and having to prioritizing emergency patients. And while there appeared to be emotional consequences for CCOT in contributing to decisions about EOL, CCOT nurses talked of job satisfaction in ensuring patients received timely EOLC and decisions. The emotional implications of their work were reported as challenging, and also appeared to affect confidence for some, especially more junior CCOT staff. Moreover, there was a sense of CCOT interventions being viewed negatively by some parent medical teams, in light of their perceived role as being the ones to raise treatment escalation decisions, or to prompt discussion regarding overall treatment goals, rather than facilitate immediate access to ICU as might be expected.

This article is protected by copyright. All rights reserved. 
The manoeuvring and management behind the scenes by CCOT to ensure that escalation plans were addressed and EOLC transitions were accomplished, seemed to incur personal emotional cost to CCOT. Each of the site's nurses referred to themselves in varying ways as being perceived as a 'Grim Reaper' or 'Angel of Death', and by raising the issue that a limitation of treatment discussion was needed, and that in some cases, the patient was likely to die in that care episode.

"I'd say that you're often seen as being though you're negative-and the grim reaper I do feel like we are seen that way, why would you raise it we're not even at that stage or anywhere near that stage, need to reiterate that you need to make a plan for out of hours..weekends."

[CCOT nurse 11, formal interview, site 3, Dec 14]

Some of the CCOT nurses appeared to have greater resilience; however, for the more junior CCOT nurses this was an uncomfortable feeling; having to counter oncologists' sometimes misplaced optimism with negativity and realism. There was individual discretion as to whether the CCOT nurses should remain involved or not, once an EOL transition was agreed; no protocols existed. This again appeared to be an interpersonal and relational issue, beyond professional curiosity to personal curiosity; one nurse hesitated to call it:

"curiosity, but not morbid curiosity, [I've] felt quite responsible as we were not going to admit him [to CCU].' [Informal interview, CCOT nurse 14, Site 1, Oct '14]

CCOT nurses seemed to developed covenants of care with long-standing patients often, which shaped interactions with both teams and families, and related to the advocacy role, previously outlined. CCOT staff would, on occasion, spend many hours with one patient ensuring the right

This article is protected by copyright. All rights reserved. 
decision was reached, having got to know the family and patient wishes, and trying to convey these to critical care colleagues, and to parent medical teams who were not always accessible. Where decisions for these patients were not reached in a timely manner, or were at odds with CCOT opinion, and in particular where this subsequently impact on the ability to provide EOLC, there was evident moral dissonance.

\section{Discussion}

The findings from this ethnography fall into the three broad themes: CCOT role in early decision-

making, Communication and EOL care. The point of uncertainty, when decisions about goals of care are first considered, presents an early opportunity for open discussion about goals of care.

Lofland raised the concept of modern dying trajectories in the 1970s (Lofland, 1978), and the work still holds true: suggesting that uncertainty diminishes the opportunity for timely and appropriate transition to end of life. 'Disproportionate' care has been used to describe the term for patients given levels of critical care support beyond what is ethically and morally justifiable, leading to family, patient and staff distress (Kompanje et al, 2013). Parent medical teams, responsible overall for patient's treatment decisions, often seemed willing to take expert input from experienced critical care outreach nurses. Where reluctance existed, this appeared to cause moral dissonance for CCOT, a feeling of CCOT not being able to do what they felt was morally right.

Uncertainty regarding underlying disease was compounded by the intercurrent critical illness. The finding is, to some degree, unique to this population, all of whom had cancer and this element of uncertainty has been termed 'dual prognostication' (Pattison et al, 2013). There is an impetus to focus on better decision-making in chronic illness, and to include patient and families' perspectives much more (Thorne and Paterson, 2003; Morton et al 2010; Legare et al 2012). The cloud of

This article is protected by copyright. All rights reserved. 
uncertainty underlying many patients' conditions seems to limits decisive action, and can lead to an apparent limbo. Recognition of clinical uncertainty, suboptimal care, and unclear communication is stressful, creates conflict and is damaging for clinical teams (Barnes et al, 2012; Bernacki et al, 2014; Pattison et al 2015). Moreover, this limbo offers little opportunity for families, patients and staff to prepare for death. CCOT may have wanted to have clarity in order to manage their discomfort in the face of clinical uncertainty. ССOT had to negotiate a fine balance of delivering enough information, being truthful but not alarmist. This negotiating process frequently led to professional tensions, which made these difficult discussions around transitions even harder.

Communicating these issues to staff, or even patients at these critical, emotional touchpoints was, at times, difficult at micro, the individual, macro, the team interactions, and macro, the organizational and professional facilitators for communication. Increasing emphasis on a healthcare culture open to challenge, where decisions are transparent and challenges and discussion regarding clinical decisions are encouraged, offers hope for the future (The Stationery Office, 2013). Issues related to DNACPR decisions surfaced throughout the ethnography, and despite measures to prompt early discussion of resuscitation decisions, it was evident that, in practice, these are tools that do not address the underlying culture of the institution or medical profession. As seen here in this research, openness was not always evident in the culture with challenges perceived to be threatening or unwarranted. Emerging evidence for care bundles and treatment escalation plans attests to this (Currow and Higginson, 2013; Etkind et al, 2015; Mockford et al 2015). These tools go some way to providing prompts, but fall short of adequately addressing how to facilitate those transitions, addressing preferences early on and ensuring timely conversations. Many of the CCOT interventions observed resulted in proportionate and timely decisions, demonstrating real impact on decisions regarding patients' trajectories and illness pathways.

This article is protected by copyright. All rights reserved. 
The cultural context of the hospital in which the teams work has a significant effect on their interactions and agency. Where they encountered conflict or reported feeling unsupported by the wider medical teams, they appeared to feel disempowered. The implications for CCOT, as a predominately lone practitioner workforce, are significant, both professionally and emotionally. Knowledge, confidence, technical competence and experience were necessary for nurses to have trust in their own practice in communicating end-of-life transitions, as well as conferring clinical credibility with parent medical teams; this emphasizes professional, meso level, and individual, micro level, elements that influence and affect agency. There was an undercurrent of the tension between doctors and nurses at times, but this was countered by evident respect, particularly from junior doctors towards CCOT, in other encounters. However, no clear conclusions on this tension could be drawn from the data we observed, and more observation may have elucidated this potential phenomenon. It was clear that raising early discussions could lead to conflict. Managing conflict has previously been identified as important in CCOT roles (Pattison and Eastman, 2012; Pattison et al, 2015). Creating mechanisms for ensuring an open culture, receptive to challenge is an important area to develop.

Seymour (2000) in her ethnography of patients dying in critical care, outlined how trust between families and staff was integral to creating a situation where a 'good death' could occur. In our study, trust was lacking where conflict existed, particularly where medical hierarchy and senior doctors were difficult to access, leading to potential delay in critical time-sensitive decisions and EOL transitions. As seen, parent medical teams had final responsibility for goals of treatment and admission decisions, and for this reason it was difficult to embrace a shared-care approach, as seen in Australia, where medical emergency teams contributed to or even led decisions (Calzavacca et al 2010). Forming trust was part and parcel of CCOT teams' day to day existence; on the one hand they were valued but had to face constant confrontation in raising questions about intent of treatment.

This article is protected by copyright. All rights reserved. 
Questioning goals of care could be perceived as a threat to trust. It raises the notion of emotional labour. James' (1989) notion of emotional labour, applied to nursing, relates to how nurses think they should feel and how they actually feel when dealing with emotionally challenging situations (Kelly et al, 2000; Savage and Moore, 2004). Dealing with dying patients might at first seem at odds with CCOT nurses' roles, whose focus historically has been on deteriorating, at-risk patients with potentially reversible illness. Identifying reversible illness is a critical component of their role, and a significant skill that comes with experience. This skill enables CCOT to diagnose dying, but has been hitherto unrecognised in their role competencies (National Outreach Forum, 2012). These nurses make qualitative assessments of risks, in relation to critical care admission and dying, on a daily basis. The unique skill set of CCOT has evolved over time to encompass this element of risk assessment and identifying reversible illness and dying, but also to manage dealing with uncertainty and to be able to respond to an ever-changing work environment and workload, in a lone capacity.

The uncontrollable factor that has emerged throughout this study relates to the parent medical team, who has to make the ultimate treatment decisions on patients. A broader focus on the wider team rather than solely through the lens of critical care outreach would have yielded even richer data and a more complete picture. Emotional labour in medicine has been explored, with doctors equally experiencing emotional dissonance when having to make decisions about patients' treatment (Sorenson and ledema, 2009; Kerasidou and Horn, 2016). Lofland (1978) outlines how, in the dying trajectory, critical illness challenges our presumptions about what should be a 'natural' death; the rapid diagnosis of dying, and early transition to end of life. Returning to the concept of uncertainty, this therefore means prolongation of this trajectory and opportunities for timely endof-life care are diminished. In the face of critical illness, there is negotiation to be had in realising the limits of what CCOT can do and critical care admission will achieve for that patient. Slomka (1992) outlined how negotiating death fulfils a need for doctors, patients and families to reconcile to the 
limits of technology. This negotiation belies an underlying issue, that of spheres of power within teams (Slomka, 1992), as witnessed here. Shared approaches dissipate the overall responsibility (Johnson et al 2000), therefore minimising dissonance, and subsequent moral distress.

Limitations

Despite taking care to delineate biases throughout, via research diaries and reflective supervision, these undoubtedly shaped the ethnographic process and findings. Furthermore, the focus was on critical care outreach and a broader lens would have captured how critical care teams fit in the context of other care teams (Hammersley and Atkinson,2007; Murphy and Dingwall, 2007) and an objectivity-subjectivity tension had to be negotiated throughout. We also may have missed the full extent of doctor-nurse tensions, although observed tentatively no clear conclusions could be drawn on this and further observations may have yielded more data in regards to this. Observer presence, may have affected team behavior (O'Reilly, 2003). Transferability is limited due to sample size, and focused hospital settings (Van der Geest and Finkler, 2004), however, core principles from the findings can be considered in a wider context, as a 'moderatum generalisation' (Payne and Williams, 2005).

\section{Implications for practice}

This research has yielded several practice recommendations. First, the need to foster a culture that is open to discussion and challenge (The Stationery Office, 2013). Second, explicit expert competencies are required for CCOT to provide person-centred care, addressing treatment and endof-life preferences. Third, development of an outline for specific organizational models of CCOT practice, to facilitate ward engagement, supporting staff who might struggle working as lone, autonomous practitioners. Developing emotional resilience is another important recommendation

This article is protected by copyright. All rights reserved. 
to support this work, based on the findings around emotional implications and consequences for individuals, through workshops and educational programmes that teach leadership and self-coping, two proposed ways of building resilience (McAlister and McKinnon, 2009). It is also important to note that CCOT is increasingly multi-professional and these issues are not limited to nurses, but also faced by physicians and physiotherapists who also work in CCOT.

\section{Conclusion}

Ethnographic data are presented which suggest that there is a negotiation, to achieve a smooth transition to end of life for individual patients, between critical care outreach, who are called when the patient is critically ill, the parent medical team and the ward-based team. Critical care outreach teams have a varied and challenging role, and a seemingly short episode of CCOT intervention can have a broad impact on the patient's pathway and affect timeliness of decisions to limit medical treatment and, where appropriate, transition to end-of-life care. The emotional consequences for CCOT nurses dealing with these transitions should not be underestimated.

\section{Relevance to clinical practice}

This research offers a useful insight into the complexity decision-making, the influence of care and team models designed to enhance patient care. It yields several practice implications, some requiring policy and cultural change, and others that could be applied at a local, meso and micro (individual) level. Nurses on the ward and CCOT nurses should feel confident in being able to challenge treatment, care and admission decisions with medical colleagues. This requires an open culture, where it is considered normal to challenge decisions, and discussion is encouraged in order

This article is protected by copyright. All rights reserved. 
to rationalise decision-making. Several factors are needed for this, such as confidence to raise concerns; developing self-efficacy and resilience techniques. There should also be professional development of CCOT, through creation of explicit expert competencies to allow CCOT to raise and, where appropriate, to address treatment and end-of-life preferences. This research, emphasising others work, suggests that with support this is a role CCOT can fulfil.

What does this paper contribute to wider clinical practice?

- There is a cautious and fine line to be trod between appreciating and respecting the patient's wishes and dealing with medical uncertainty in the face of critical illness, superimposed upon an underlying chronic or acute diagnosis.

- There are challenges in identifying the optimal point for CCOT involvement, and subsequent transitions, and in sustaining CCOT involvement when there are multiple pathologies (e.g. sepsis in a dying patient with advanced disease).

- This research shows how CCOT nurses can take lead roles in early and open discussion about treatment goals, but only if supported in those roles by all the 'parent' teams involved. This emphasises an undercurrent of power seen in the data, and the issues of confidence and requisite experience to take on such roles.

- The importance of support suggests that if the organisational systems and teams are not aligned, then the CCOT nurses are likely to experience emotional consequences, which could perhaps have been avoided.

This article is protected by copyright. All rights reserved. 
Declarations

The authors have no conflicts of interest to declare. This study was funded by the General Nursing Council Trust.

\section{References}

Aslakson R. 2015. Time-Limited Trials in the ICU: Seeing the Forest Beyond the Bark and Trees. Critical care medicine. 43(12):2676-8

Bardsley M, Georghiou T, Spence R, Billings J. 2016. Factors associated with variation in hospital use at the end of life in England. BMJ Support Palliat Care. 2016 Mar 24. pii: bmjspcare-2015-000936. doi: 10.1136/bmjspcare-2015-000936. [Epub ahead of print]

Barnes S, Gardiner C, Gott M, Payne S, Chady B, Small N, Seamark D, Halpin D. 2012. Enhancing patient-professional communication about end-of-life issues in life-limiting conditions: a critical review of the literature. J Pain Symptom Manage. 44(6):866-79.

Bennett MI, Ziegler L, Allsop M, Daniel S, Hurlow A. 2016. What determines duration of palliative care before death for patients with advanced disease? A retrospective cohort study of community and hospital palliative care provision in a large UK city. BMJ Open; 6(12):e012576.

Bernacki RE, Block SD, American College of Physicians High Value Care Task Force. 2014.

Communication about serious illness care goals: a review and synthesis of best practices. JAMA Intern Med. 174(12):1994-2003

Bloor M. 1978. On the analysis of observational data: a discussion of the worth and uses of inductive techniques and respondent validation. Sociology 12:545-52.

Bristowe K, Carey I, Hopper A, Shouls S, Prentice W, Caulkin R, Higginson IJ, Koffman J. 2015. Patient and carer experiences of clinical uncertainty and deterioration, in the face of limited reversibility: $A$ comparative observational study of the AMBER care bundle. Palliative Medicine, 29(9), 797-807

This article is protected by copyright. All rights reserved. 
Calzavacca P, Licari E, Tee A, Egi M, Downey A, Quach J, Haase-Fielitz A, Haase, M, Bellomo, R. 2010. The impact of Rapid Response System on delayed emergency team activation patient characteristics and outcomes--a follow-up study. Resuscitation 81(1):31-5.

Coombs MA, Nelson K, Psirides AJ, Suter N, Pedersen A. 2016. Characteristics and dying trajectories of adult hospital patients from acute care wards who die following review by the rapid response team. Anaesth Intensive Care. 44(2):262-9.

Currow DC, Higginson I. 2013. Time for a prospective study to evaluate the Amber Care Bundle. BMJ Support Palliat Care. 3(4):376-7

Dahmen BM, Vollmann J, Nadolny S, Schildmann J. 2017. Limiting treatment and shortening of life: data from a cross-sectional survey in Germany on frequencies, determinants and patients' involvement. BMC Palliative Care. 16(1):3.

Department of Health. 2000. Comprehensive critical care. London: Department of Health

Department of Health. 2009. End of life Care Programme. London: Department of Health

Emerson RM, Fretz RI, Shaw LL. 2001. Participant Observation and Fieldnotes. In Atkinson P, Coffey A, Delamont S, Lofland J, Lofland L. (Eds.), Handbook of Ethnography. Thousand Oaks, CA: Sage Publications. pp: 351-368.

Etkind SN, Karno J, Edmonds PM, Carey I, Murtagh FE. 2015. Supporting patients with uncertain recovery: the use of the AMBER care bundle in an acute hospital. BMJ Support Palliat Care. 5(1):95-8

Fisher M, Ridley S. 2012. Uncertainty in end-of-life care and shared decision-making. Critical Care and Resuscitation 14(1):81-7

Frost DW, Cook DJ, Heyland DK, Fowler RA. 2011. Patient and healthcare professional factors influencing end-of-life decision-making during critical illness: a systematic review. Crit Care Med. 39(5):1174-89

This article is protected by copyright. All rights reserved. 
Gold Standards Framework. 2018. Gold Standards Framework Advanced Care Planning (Available at: http://www.goldstandardsframework.org.uk/advance-care-planning. Accessed 27.1.18)

Hammersley M, Atkinson P. 2007. Ethnography: Principles in Practice 3rd Edition. Taylor and Francis

Higginson IJ, Rumble C, Shipman C, Koffman J, Sleeman KE, Morgan M, Hopkins P, Noble J, Bernal W, Leonard S, Dampier O, Prentice W, Burman R, Costantini M. 2016. The value of uncertainty in critical illness? An ethnographic study of patterns and conflicts in care and decision-making trajectories. BMC Anesthesiol. 16:11. doi: 10.1186/s12871-016-0177-2.

Hua M, Halpern SD, Gabler NB, Wunsch H. 2016. Effect of ICU Strain on Timing of Limitations in LifeSustaining Therapy and Death. Intensive Care Medicine.42(6):987-94.

Hui D, Kim SH, Kwon JH, Tanco KC, Zhang T, Kang JH Rhondali W, Chisholm G, Bruera E. 2012. Access to palliative care among patients treated at a comprehensive cancer center. Oncologist;

17(12):1574-1580

James, N. 1989. Emotional labour: skill and work in the social regulation of feelings. The Sociological Review, 37:15-42.

Johnson N, Cook D, Giacomini M, Willms, D. 2000. 'Towards a 'Good' Death: End-of-Life Narratives Constructed in an Intensive Care Unit.' Culture, Medicine and Psychiatry 24:275-295

Jones DA, Bagshaw SM, Barrett J, Bellormo R, Bhatia G, Bucknall TK, Casamento AJ, Duke GJ, Gibney N, Hart G., Hillman KM, Jaderling G, Parmar A, Parr MJ. 2012. The role of the medical emergency team in end-of-life care: A multicenter, prospective, observational study*. Critical Care Medicine 40(1):98-103

Kelly D, Ross S, Gray B, Smith P. 2000. Death, dying and emotional labour: problematic dimensions of the bone marrow transplant nursing role? J Adv Nurs. 32(4):952-60.

Kerasidou A Horn R. 2016. Making space for empathy: supporting doctors in the emotional labour of clinical care. BMC Medical Ethics. 2016 17:8 doi.org/10.1186/s12910-016-0091-7

This article is protected by copyright. All rights reserved. 
Kompanje EJ, Piers RD, Benoit DD. 2013. Causes and consequences of disproportionate care in intensive care medicine. Curr Opin Crit Care. 19(6):630-5.

Legare F, Turcotte S, Stacey D, Ratte S, Kryworuchko J, Graham ID. 2012. Patients' perceptions of sharing in decisions: a systematic review of interventions to enhance shared decision making in routine clinical practice. Patient. 5:1-19.

Lincoln YS Guba E. 1985. Naturalistic Inquiry. London: Sage

Lofland L. 1978. The craft of dying: The modern face of death. Beverly Hills: Sage

McAlister M, McKinnon J. 2009. The importance of teaching and learning resilience in the health disciplines: A critical review of the literature. Nurse Education Today. 29:371-379

Mockford C, Fritz Z, George R, Court R, Grove A, Clarke B, Field R, Perkins GD. 2015. Do Not Attempt Cardiopulmonary Resuscitation (DNACPR) orders : a systematic review of the barriers and facilitators of decision making and implementations. Resuscitation, 88:99-113

Morton RL, Tong A, Howard K, Snelling P, Webster AC. 2010. The views of patients and carers in treatment decision making for chronic kidney disease: systematic review and thematic synthesis of qualitative studies BMJ; 19;340:c112. doi: 10.1136/bmj.c112.

Murphy E, Dingwall R. 2007. Informed consent, anticipatory regulation and ethnographic practice. Soc Sci Med. 65(11):2223-34.

National Confidential Enquiry into Patient Outcome and Death (NCEPOD) (2008) Systemic AntiCancer Therapy: for better, for worse? Available at: http://www.ncepod.org.uk/2008sact.htm (Accessed Sep 10th 2017)

National Outreach Forum. 2012. Operational Standards and Competencies for Critical Care Outreach Services. Available at: http://www.norf.org.uk/NOrF_news?mode=PostView\&bmi=858315 (Accessed April 10th 2017)

This article is protected by copyright. All rights reserved. 
O'Reilly K. 2009. Key Concepts in Ethnography. London: Sage.

Pattison N, Ashley S, Farquhar-Smith P, Roskelly L, O'Gara G. 2010. Thirty-day mortality in critical care outreach patients with cancer: an investigative study of predictive factors related to outreach referral episodes. Resuscitation 81(12): 670-5

Pattison N, Carr S, Turnock C, Dolan S. 2013. Viewing in slow motion: end-of-life care in critically ill cancer patients. Journal of Clinical Nursing 22(9-10): 1442-54.

Pattison N, O'Gara, Wigmore T. 2015. End-of-Life decisions and Critical Care Outreach: a mixed method study. American Journal of Critical Care. 24(3):232-40.

Pattison, N., Eastman, E. 2012. 'A mixed method study of critical care outreach referrals.' Nursing in Critical Care 17(2):71-82

Payne G, Williams M. 2005. Generalization in Qualitative Research. Sociology. 39:2295-314

Savage J, Moore L. 2004. Interpreting accountability. An ethnographic study of practice nurses, accountability and multidisciplinary team decision-making in the context of clinical governance. RCN Institute: Available at : http://www.rcn.org.uk/_data/assets/pdf_file/0008/78605/002249.pdf (accessed 3rd Mar 2017)

Seymour JE. 1999. Revisiting medicalisation and 'natural' death. Social Science and Medicine 49:691704

Seymour, J.E. 2000. "Negotiating natural death in intensive care." Social Science and Medicine 51(8):1241-52

Slomka J. 1992. 'The negotiation of death: clinical decision-making at the end of life.' Social Science and Medicine 35 pp251-259

This article is protected by copyright. All rights reserved. 
Sorensen R, ledema R. 2009. Emotional labour: clinicians' attitudes to death and dying. Journal of Health Organization and Management 23(1):5-22

Tan LH, Delaney A (2014. Medical emergency teams and end-of-life care: a systematic review. Crit Care Resusc. 16(1):62-8.

The Stationery Office. 2013. Report of the Mid Staffordshire NHS Foundation Trust Public Inquiry. London: The Stationery Office.

Thorne S, Paterson B, Russell C. 2003. The structure of everyday self-care decision making in chronic illness. Qual Health Res. 13(10:1337-52.

Van der Geest S, Finkler V. 2004. Hospital ethnography: introduction. Soc Sci Med. 59(10):19952001. 
Table 1: Facet of Main Themes

\begin{tabular}{|c|c|}
\hline Sub-themes & Main theme \\
\hline $\begin{array}{l}\text { 1. Power: a) Experience level and impact on influence; } \\
\text { Ability to challenge consultants; Medical hegemony; b) } \\
\text { Receptivity of teams (palliative care/medical teams) }\end{array}$ & $\begin{array}{l}\text { Communicating end-of-life } \\
\text { transitions/ Early decision-making } \\
\text { and the role of critical care outreach }\end{array}$ \\
\hline $\begin{array}{l}\text { 2. Context of workload (self/teams): Level of CCOT } \\
\text { activity impacts on interventions/ engagement }\end{array}$ & End-of-life care and the input of CCOT \\
\hline $\begin{array}{l}\text { 3.How teams (medical/CCOT) work: structure, access } \\
\text { and respect } 7 \text {. Out of hours stressors: lone } \\
\text { practitioners } 2 \text {. Consultative model/responsive model } \\
\text { of CCOT practice }\end{array}$ & $\begin{array}{l}\text { Early decision-making and the role of } \\
\text { critical care outreach }\end{array}$ \\
\hline $\begin{array}{l}\text { 4. Emotional implications: Ione practitioner. Emotional } \\
\text { labour/reserve }\end{array}$ & End-of-life care and the input of CCOT \\
\hline 5. Culture of addressing end-of-life issues & Communicating end-of-life transitions \\
\hline 6. Managing clinical uncertainty & $\begin{array}{l}\text { Early decision-making and the role of } \\
\text { critical care outreach/ } \\
\text { Communicating end-of-life transitions }\end{array}$ \\
\hline $\begin{array}{l}\text { 7. When to be involved and when not? Threat of } \\
\text { outreach: the grim reaper/angel of death }\end{array}$ & End-of-life care and the input of CCOT \\
\hline 8. How decision-making is reached: process issues & $\begin{array}{l}\text { Early decision-making and the role of } \\
\text { critical care outreach }\end{array}$ \\
\hline
\end{tabular}

This article is protected by copyright. All rights reserved. 


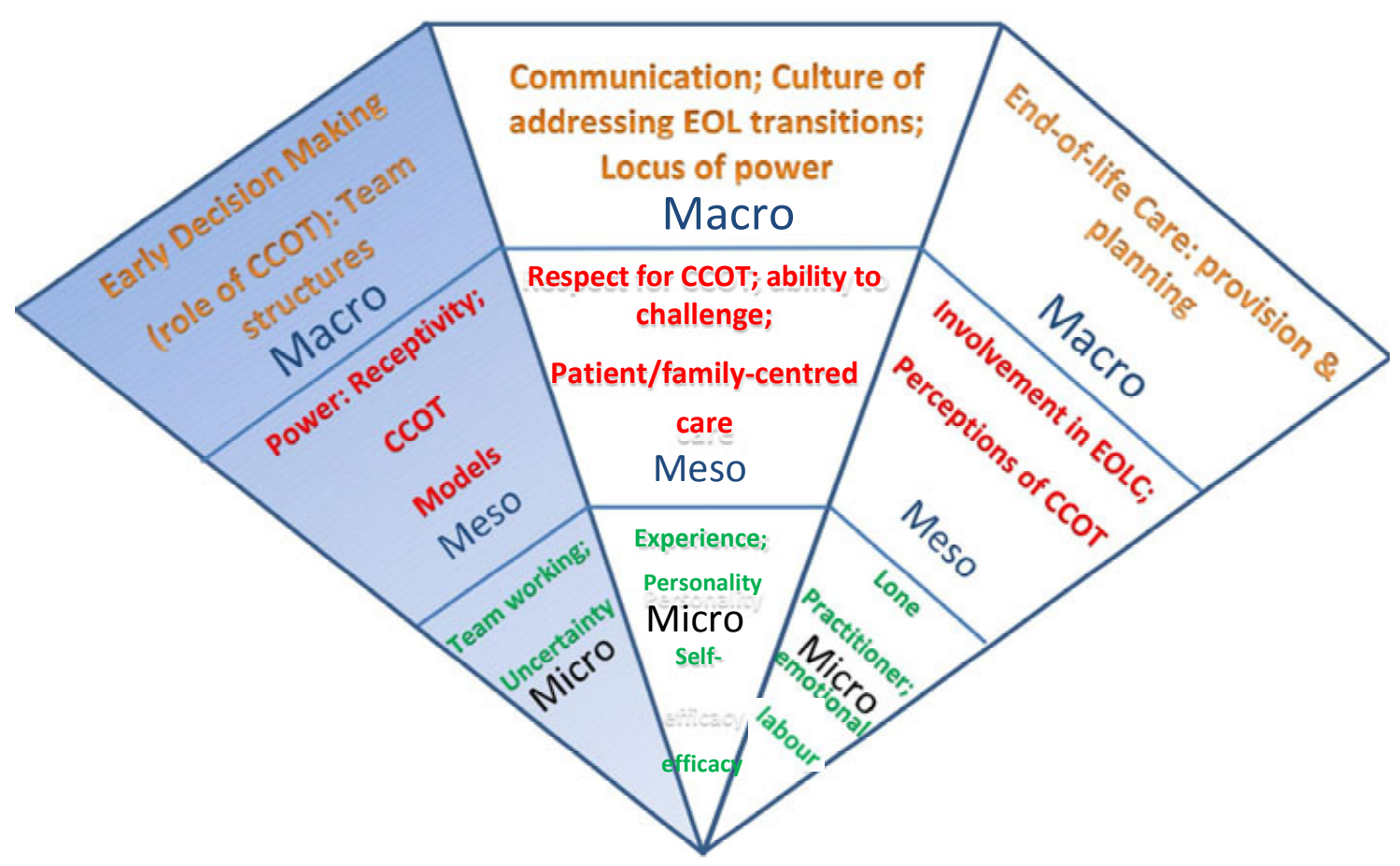

This article is protected by copyright. All rights reserved. 